\title{
Wirtschaftsinformatikstudium an Universitäten und Fachhochschulen - Konvergenz oder Differenzierung?
}

DOI 10.1007/s11576-013-0371-5

\section{Die Autoren}

Prof. Dr. Peter Loos ( $\varangle)$

Institut für Wirtschaftsinformatik im DFKI

Universität des Saarlandes

66123 Saarbrücken

Deutschland

loos@iwi.uni-sb.de

\section{Rosemarie Clarner \\ Prof. Dr. Frank Hermann \\ Prof. Dr. Thomas Hess \\ Prof. Dr. Andreas Gadatsch \\ Prof. Dr. Elmar Sinz}

Online publiziert: 2013-07-13

This article is also available in English via http://www.springerlink.com and http://www.bise-journal.org: Loos P (2013) Business and Information Systems Engineering Programs at Universities and Fachhochschulen - Convergence or Differentiation? Bus Inf Syst Eng. doi: 10.1007/s12599013-0276-2.

(C) Springer Fachmedien Wiesbaden 2013

\section{Einleitung}

Wirtschaftsinformatik wird seit den 1970er-Jahren als eigenständiges Studienfach angeboten, sowohl an Universitäten als auch an Fachhochschulen. Universitäten haben eine lange Tradition und folgen dem humboldtschen Bildungsideal, bei dem Lehre und Forschung als Einheit gesehen werden. Dagegen wurden Fachhochschulen erst in den 1960er-Jahren in Deutschland als weitere tertiäre Ausbildungsinstitutionen eingeführt und später von verschiedenen europäischen Ländern übernommen. Fachhochschulen fokussierten auf die praxisorientierte Ausbildung, sie hatten keinen Forschungsauftrag. Im Rahmen des Bologna-Prozesses, mit dem ein einheitlicher europäischer Hochschulraum mit zweistufigen Abschlüssen geschaffen werde soll, werden die sichtbaren Unterschiede zwischen den beiden Hochschultypen aufgehoben. Universitäten und Fachhochschulen haben heute gleich lange Studienprogramme, die mit einem Bachelor oder Master abschließen. Die Abschlüsse von Fachhochschulen sind formal denen der Universität gleichgestellt. Bei beiden Hochschultypen wird die Beschäftigungsfähigkeit („Employability“) der Absolventen gefordert. Ein Fachhochschul-Master berechtigt prinzipiell zur Promotion. Auch die anwendungsorientierte Forschung gehört heute zu den Aufgaben von Fachhochschulen. Die meisten Fachhochschulen haben zwischenzeitlich ihren Namen in Hochschule geändert und benutzen die englische Bezeichnung „University of Applied Science".

Dennoch gibt es weiterhin deutliche Unterschiede. Das Studium an den Fachhochschulen ist wesentlich stärker anwendungsorientiert und enthält in der Regel eine längere Praxisphase, z. B. ein Praxissemester. Studiengänge an Universitäten sind dagegen stärker theorieorientiert. Dies spiegelt sich in dem Qualifikationsweg der Hochschullehrer wider: Für Universitätsprofessoren ist er rein akademisch und umfasst, neben einer Promotion, eine Habilitation oder eine Juniorprofessur. Von einem Fachhochschulprofessor wird neben einer Promotion eine fünfjährige Berufspraxis gefordert. Weiterhin haben Fachhochschulen kein Promotionsrecht.

Welche Konsequenzen haben die Gemeinsamkeiten und Unterschiede auf die Studieninhalte von Wirtschaftsinformatik-Studiengängen an den beiden Hochschultypen? Zur Diskussion wurden neben Professoren von Universitäten und Fachhochschulen auch Persönlichkeiten aus der Wirtschaft eingeladen, die u. a. folgende Fragen erörtern sollen:

- Wie unterscheiden sich Studiengänge der Wirtschaftinformatik an Universitäten und Fachhochschulen? Welche Gemeinsamkeiten weisen sie auf?
- Wie viel Praxiswissen sollte in universitären Studiengängen, wie viel Theorie in Fachhochschulstudiengängen enthalten sein?

- Wie können sich die Wirtschaftsinformatik-Studiengänge an den unterschiedlichen Hochschultypen profilieren?

- Wie sollten sich die Studiengänge an den unterschiedlichen Hochschultypen weiterentwickeln?

- Was erwartet der Arbeitsmarkt von den Absolventen?

Die Einladung zu dieser Diskussion haben folgende Wissenschaftler und Praktiker angenommen (in alphabetischer Reihenfolge):

- Rosemarie Clarner, Scheer Group

- Prof. Dr. Andreas Gadatsch, Hochschule Bonn-Rhein-Sieg

- Prof. Dr. Frank Hermann, Hochschule Regensburg

- Prof. Dr. Thomas Hess, LMU München

- Prof. Dr. Elmar Sinz, Universität Bamberg

Aus Sicht eines mittelständischen Beratungshauses beschreibt Rosemarie Clarner, Senior Partner Human Resources bei der Scheer Group, die Anforderungen an Bewerber mit dem Abschluss Wirtschaftsinformatik. Sie stellt fest, dass sich im Zuge des Bologna-Prozesses die Unterschiede zwischen Absolventen von Fachhochschulen und Universitäten zunehmend verringern. Sie bevorzugt eine hohe Anwendungsnähe und Praxisrelevanz in der Ausbildung, die sie stärker in den Fachhochschulen umgesetzt sieht. Damit legt sie einen anderen Schwerpunkt bezüglich erwarteter Qualifikationen als bspw. Burkhard Schwenker, der ein stark theorieorientiertes Studium als Vorbereitung für die Arbeitswelt fordert (Loos et al. 2013). Im Personalbeschaffungsprozess spiele der Hochschultyp des Abschlusses aber eine untergeordnete Rolle, wichtiger sind bspw. Studienschwerpunkte, Auslands- und Praxiserfahrung sowie sonstige Qualifikationen. Insgesamt wünscht sie sich eine stärkere Vermittlung von Sozial- und Selbst- 
kompetenz im Wirtschaftsinformatikstudium.

Frank Herrmann, Sprecher des Arbeitskreises Wirtschaftsinformatik an Fachhochschulen, sieht die Umsetzung von Unternehmensprozessen mit ERPSystemen als Leitidee für viele Studienkonzepte an Fachhochschulen, auch bedingt durch die berufspraktische Voraussetzung für Fachhochschulprofessoren. Daneben sind theoretische Grundlagen und Methoden notwendig, um Anwendungskonzepte betrieblicher Systeme zu verstehen.

Thomas Hess, Sprecher der Wissenschaftlichen Kommission Wirtschaftsinformatik im Verband der Hochschullehrer für Betriebswirtschaft, sieht in der Theorieorientierung ein wichtiges Differenzierungsmerkmal universitärer Studiengänge. In unserer Disziplin, deren praktische Anwendung durch schnelle Entwicklungszyklen gekennzeichnet ist, ist das Theoriewissen stabil. Die theoretischen Grundlagen erstrecken sich dabei auf die Mutterdisziplinen Wirtschaftswissenschaften und Informatik. Aufgrund der theoretischen Breite biete sich an, selektive Grundlagen zu Beginn des Studiums zu legen und diese im weiteren Verlauf themenbezogen auszubauen.

Andreas Gadatsch vergleicht Wirtschaftsinformatik-Studiengänge an Fachhochschulen und Universitäten anhand ihres Curriculums und kommt zu dem Schluss, dass die Studiengänge an beiden Hochschultypen ähnlich aufgebaut sind. Unterschiede bestehen in der stärkeren Praxisorientierung an Fachhochschulen sowie in der stärkeren Forschungsorientierung an Universitäten. Er sieht darin weiterhin Profilierungs- und Differenzierungsmöglichkeiten und wünscht sich eine hohe Durchlässigkeit zwischen den Hochschultypen.

Elmar Sinz, der auf eine langjährige Erfahrung bei Akkreditierungsverfahren zurückblicken kann, stellt fest, dass die Studienangebote und die Möglichkeit der Studiengestaltung an beiden Hochschultypen zugenommen haben. Ähnlich wie Thomas Hess argumentiert er mit den unterschiedlichen Halbwertszeiten der Wissensinhalte unseres Fachgebietes und kommt zu dem Schluss, dass die theoretischen Grundlagen den längsten Bestand haben und somit als wichtige Studieninhalte frühzeitig im Curriculum verankert werden sollten. Nur so könne sichergestellt werden, dass sich die Absolventen auch langfristig auf neue
Herausforderungen im Berufsleben einstellen können. Weiterhin wirft er die Frage auf, warum es keinen einstufigen Master gibt, der eine ausgewogenere Studienverlaufskonzeption ermöglicht.

Zusammenfassend kann festgehalten werden, dass trotz Konvergenz der durch den Bologna-Prozess vorgegebenen Rahmenbedingungen weiterhin eine Differenzierung zwischen den Hochschultypen gesehen wird, insbesondere hinsichtlich des Praxisbezugs und der Theorieausbildung. Allerdings fällt die Bewertung, wie stark diese in einem Wirtschaftsinformatikstudium vertreten sein sollen, unterschiedlich aus. Wenn auch Sie zu diesem Thema - oder einem anderen Artikel der Zeitschrift WIRTSCHAFTSINFORMATIK - Stellung nehmen möchten, senden Sie Ihre Stellungnahme (max. 2 Seiten) bitte an loos@iwi.uni-sb.de.

Prof. Dr. Peter Loos Institut für Wirtschaftsinformatik im DFKI

Universität des Saarlandes, Saarbrücken

\section{Wie unterscheiden sich Studiengänge der Wirtschaftsinformatik an Universität und Fachhochschule?}

Als Beratungsunternehmen mit Fokus Geschäftsprozessmanagement suchen wir Kandidaten mit einem Abschluss in Wirtschaftsinformatik. Dieses Fach wird sowohl an Universitäten als auch Fachhochschulen und dualen Hochschulen angeboten. Es wird immer wieder die Frage an mich herangetragen, von welcher Hochschule wir am liebsten Kandidaten rekrutieren. Dies lässt sich so pauschal nicht beantworten. Hierzu muss man sich die Unterschiede zwischen den verschiedenen Hochschultypen anschauen. Im Folgenden möchte ich insbesondere auf die Unterschiede zwischen Universität und Fachhochschule eingehen.

Universitäten bilden als Leitidee für die Wissenschaft aus, obwohl nur wenige $\mathrm{Ab}$ solventen die Wissenschaft als Beruf wählen. Die Domäne der Fachhochschulen ist die praxisbezogene Lehre. So war es bisher. Ganz so trennscharf sind die Unterschiede allerdings nicht mehr. Im $\mathrm{Zu}$ ge der Bologna Reform bilden auch Universitäten berufsbezogener aus und Fachhochschulen machen sich mit anwendungsorientierter Forschung einen Na- men. Universitäten und Fachhochschulen vergeben formal die gleichen Abschlüsse, Bachelor und Master.

Unterschiede existieren allerdings immer noch. Bei den Fachhochschulen liegt der Schwerpunkt noch immer mehr auf der Anwendung, obwohl an fast allen Fachhochschulen auch angewandte Forschung betrieben wird. Es wird mehr Wert auf die Praxisrelevanz gelegt, d. h. auf die Umsetzung der Theorie in die Praxis, und mehr Zeit mit Praxisprojekten und Gruppenarbeit verbracht. Dies wird durch entsprechende Praxissemester und Abschlussarbeiten in der Wirtschaft ergänzt. Der Kontakt zur Wirtschaft ist insgesamt stärker ausgeprägt. Dies geschieht auch durch das Profil des Lehrpersonals. Dozenten an der Fachhochschule müssen mindestens 5 Jahre hauptberuflich gearbeitet haben, davon 3 Jahre außerhalb der Hochschule. Dadurch haben sie mehr Praxiserfahrung, insbesondere auch in der Koordination von Projekten und im Projektmanagement. An Universitäten gilt diese Voraussetzung nicht. Hier können Doktoranden nach ihrer Promotion direkt mit der Lehrtätigkeit beginnen.

Viele Fachbereiche an Fachhochschulen arbeiten an Themen, die in der Wirtschaft gerade gefragt sind. Dafür pflegen sie enge Kooperationen zu Unternehmen. Es gibt Fachhochschulen, die Dependancen an den Sitz von Unternehmen ausgliedern, um effizienter Projekte, Bachelor- oder Masterarbeiten unterstützen zu können. Fachhochschulen entwickeln in stärkerem Maße neuartige Kooperationsmodelle; an Universitäten ist dies eher die Ausnahme. Sie verharren häufig noch in ihrem Campuscharakter. Diese Flexibilität zahlt sich für Fachhochschulen aus. Das bedeutet aber auch, dass der Anteil von theoretischem Wissen, das vermittelt wird, geringer bemessen ist als im Rahmen eines universitären Studiums. Allgemein kann man sagen, dass an Universitäten ein Drittel Praxis vermittelt wird, an Fachhochschulen zwei Drittel, bei der Vermittlung der Theorie verhält es sich dann umgekehrt.

Fachhochschulen orientieren sich thematisch und organisatorisch insgesamt mehr an den Bedarfen der Wirtschaft. Dies zeigt sich auch darin, dass schneller spezialisierte Studiengänge entstehen und genauso schnell auch wieder abgeschafft werden.

An der klassischen Universität wird in der Lehre immer noch meist das vermittelt, was später für die Forschung benö- 
tigt wird: Grundlagenwissen und Theorie. Ergänzt wird dies durch Beispiele aus der Praxis, die die Theorie bestätigen. Eine stärkere Kooperation zwischen Universitäten und Wirtschaft wäre wünschenswert.

Es wäre sicher sinnvoll, wenn auch soziale und Selbstkompetenzen vermittelt würden. Denn sie werden in der Praxis benötigt und gehören zu den Anforderungskriterien in der Wirtschaft. Es geht beispielsweise um die Fähigkeit, mit Anderen in Projektteams konstruktiv und effektiv zusammenzuarbeiten, ebenso um kommunikative Fähigkeiten. Es kommt darauf an, zielgruppengerecht zu kommunizieren, das Auftreten und die Umgangsformen professionell an der Zielgruppe und dem Anlass auszurichten, zuzuhören, Sensitivität für die Signale des Gesprächspartners zu entwickeln. Wir benötigen Menschen, die zu konstruktiver Selbstreflexion in der Lage und bereit sind, ein Arbeitsleben lang $\mathrm{zu}$ lernen und sich weiterzuentwickeln. Menschen, die in der Wirtschaft gesucht werden, können Konflikte bewältigen, sind lösungs- und zielorientiert, $\mathrm{zu}$ einem partnerschaftlichen und toleranten Diskurs von Meinungsverschiedenheiten sowie zu einer aktiven Integration von Interessengegensätzen und Wertediskrepanzen in der Lage. Sie können präsentieren, Verhandlungen führen, beherrschen die entsprechenden Techniken und überzeugen in ihrer Argumentation. Die Grundlagen dafür könnten hervorragend im Rahmen des Studiums geschaffen werden.

Beide Hochschularten haben sich der Internationalisierung verschrieben, wobei hier noch ein leichter Vorteil bei den Universitäten liegt. Jedoch profilieren sich auch Fachhochschulen immer mehr durch internationale Kooperationen und Studienprogramme. Auch bei studienbezogenen Auslandsaufenthalten und fremdsprachlichen Kompetenzen holen Fachhochschulen immer mehr auf. Allerdings liegen bei Auslandsaufenthalten die Universitäten noch leicht vorne, vielleicht, weil es aufgrund der freieren Studiengestaltung leichter möglich ist, einen Auslandsaufenthalt einzubauen.

Sowohl Universitäten als auch Fachhochschulen sollten durchaus die Schwerpunkte ihrer Profile beibehalten, sich jedoch auch annähern. Es liegen Kooperationsmöglichkeiten nahe, um sowohl die jeweiligen Stärken zu nutzen als auch die Schwächen auszugleichen. Hier stehen allerdings häufig noch Statusargumente entgegen; man betrachte nur das konfliktbehaftete Thema um das Promotionsrecht an Fachhochschulen. Diese könnten meines Erachtens durch innovative Kooperationsmodelle ausgeräumt werden.

Insgesamt lässt sich sagen, dass wir gar nicht so sehr darauf schauen, ob ein Kandidat von der Universität oder Fachhochschule kommt, sondern welche Studienschwerpunkte und Qualifikationen er vorweisen kann, ob er einen Bacheloroder Masterabschluss hat, über den Tellerrand hinausgeschaut hat durch Praktika und Auslandsaufenthalte und ob er die persönlichen Skills mitbringt, die gefordert werden. Hier sind die bereits beschriebenen Fähigkeiten ebenso gefragt wie Kreativität, Flexibilität und sehr gute Englischkenntnisse. Auch in der Vergütung machen wir keine Unterschiede mehr zwischen Fachhochschul- und Universitätsabsolventen.

Rosemarie Clarner Scheer Group

\section{Wirtschaftsinformatik an Fachhochschulen - Praxis, Theorie und Beschäftigungsfähigkeit}

Der erste vollzügige Studiengang für Wirtschaftsinformatik wurde 1971 an der Fachhochschule Furtwangen gegründet. Entsprechend den einschlägigen Empfehlungen der Gesellschaft für Informatik realisieren die Studiengänge für Wirtschaftsinformatik das so genannte Dreisäulenmodell mit Veranstaltungen zur Informatik, zur originären Wirtschaftsinformatik und zur Betriebswirtschaftslehre, und zwar meiner Wahrnehmung nach sowohl an Fachhochschulen als auch an Universitäten, sicher auch verstärkt dadurch, dass oftmals, inoffiziell, bei den Akkreditierungen dieser Studiengänge diese Empfehlungen berücksichtigt werden. Bedingt durch die Akkreditierungsvorschriften werden $\mathrm{zu}$ allen drei Stoffgebieten fachspezifische Module mit einzelnen Lehrveranstaltungen angegeben.

Die Leitidee vieler Konzepte lässt sich durch die Unterstützung von Prozessen durch Informationssysteme zusammenfassen. Die Durchdringung vieler Unternehmen mit ERP-Systemen (EnterpriseResource-Planning), vor allem dem der SAP AG, verstärkt diese Tendenz nachhaltig. Dieser Einfluss dürfte an Fachhochschulen aufgrund einer mindestens fünfjährigen beruflichen Tätigkeit als Berufungsvoraussetzung für Hochschullehrer relativ hoch sein. Ein weiterer Beleg ist die sehr hohe Verfügbarkeit des SAP-Systems an Universitäten und Fachhochschulen; siehe die Angaben des entsprechenden Programms der SAP (SAP University Alliances) im Internet. Jede der mir bekannten Universitäten nutzt ein SAP-System in der Lehre. Viele Lehrenden dürfte die Relevanz ihrer einzelnen fachspezifischen Module durch den Lebenszyklus eines Informationssystems, vor allem eines ERP-Systems, rechtfertigen. Es handelt sich um die Phasen Entdeckung und Evaluierung, Implementierung, Produktivschaltung („Go Live“), Betrieb und kontinuierliche Verbesserung - danach dürften sich diese Phasen wiederholen. In der Implementierungsphase sind die funktionalen Anforderungen wie Einstellung von Parametern durchzuführen, die Konfiguration von Verfahren vorzunehmen, funktionale Erweiterungen, auch von Verfahren, sowie Schnittstellen $\mathrm{zu}$ implementieren. Demgegenüber sind im Betrieb und der kontinuierlichen Verbesserung technische Probleme im Anwendungsbetrieb zu lösen, die Laufzeit zu verringern, die Effizienz des Systembetriebs zu erhöhen, die Integration (vor allem im Hinblick auf die Datenkonsistenz) zu erhöhen und die Hochverfügbarkeit sicherzustellen. Eine Verallgemeinerung dieser Themen bilden die Kapitel in Standardlehrbüchern zur Wirtschaftsinformatik, siehe das besonders anerkannte Buch Laudon und Laudon (2002) und seine Übersetzung ins Deutsche (Laudon et al. 2009). Diese Ausrichtung dürfte einen wesentlichen Beitrag zu den überdurchschnittlich guten Berufsaussichten von Wirtschaftsinformatikern leisten.

Die oben genannten drei Säulen, die in der Regel den nahezu gleichen Umfang haben, determinieren die gelehrten Methoden. Nicht nur wegen zeitlicher Restriktionen, sondern auch wegen der oben angesprochenen Leitidee erfolgt im Rahmen der Informatiksäule eine weniger tiefe Ausbildung in Mathematik und angewandter Logik als in den Studiengängen zur Allgemeinen Informatik. Dass ein Theoriebezug zur Umsetzung der oben genannten Leitidee notwendig ist, zeigt exemplarisch der Peitscheneffekt im Supply-Chain-Management. Beim Peitscheneffekt neigen Bestellungen beim Lieferanten zu größeren Schwankungen als Verkäufe an den Kunden und weichen damit von der Nachfrage ab, und diese Abweichung schaukelt sich in 
die vorgelagerte Richtung der Lieferkette auf. Durch einen verbesserten Austausch von Informationen zwischen den beteiligten Unternehmen in der logistischen Kette kann der Peitscheneffekt signifikant reduziert und sogar marginalisiert werden. Die (anspruchsvolle) wissenschaftliche Erklärung ist notwendig, um das Potenzial zusätzlicher Information $\mathrm{zu}$ beweisen, und Informationstechnologie ist notwendig, um diese bereit zu stellen. Viele WirtschaftsinformatikStudiengänge an Fachhochschulen integrieren daher Modelle zur Erklärung solcher Konzepte.

Bereits seit mehreren Jahren findet im Rahmen der Jahrestagung des Arbeitskreises Wirtschaftsinformatik an Fachhochschulen eine Tagung statt, in der Hochschullehrer (von Fachhochschulen) ihre aktuellen Forschungsarbeiten präsentieren. Eine Durchsicht zeigt, dass praktische Erfahrungen, Konstruktionsund Implementierungsarbeiten, gerade für ERP-Systeme, eine zentrale Rolle spielen und so Forschungsmethoden der praktischen Informatik dominieren. Neben dieser gestalterischen Forschung werden Erklärungen empirisch, vielfach per Simulationen, publiziert. Werden Probleme aus etablierten Gebieten wie beispielsweise dem Operations-Research behandelt, so werden die dort üblichen Forschungsmethoden verwandt. In der Regel wird die dafür erforderliche Methodik in den einschlägigen Masterveranstaltungen eingeübt.

Professor Dr. Frank Herrmann Hochschule Regensburg

\section{Theorieelemente in universitären Wirtschaftsinformatik- Studiengängen}

Das Hochschulsystem im deutschsprachigen Raum hat sich in den letzten Jahren und Jahrzehnten stark ausdifferenziert. Neben die Universitäten sind Fachhochschulen und auch Berufsakademien getreten. Dies hat seinen Niederschlag auch in der Wirtschaftsinformatik (WI) gefunden. WI-Studiengänge finden sich mittlerweile in allen drei genannten Hochschultypen. Eine derartige Differenzierung macht langfristig allerdings nur Sinn, wenn die Studiengänge unterschiedliche Ziele verfolgen bzw. unterschiedliche Zielgruppen adressieren. Ein wichtiges Differenzierungsmerkmal universitärer Studiengänge soll die Theorieorientierung sein. Wie für jedes Fach, stellt sich auch für die WI die Frage, wie diese Theorieorientierung konkret aussehen kann.

Zunächst stellt sich die Frage nach dem warum, konkret: Warum soll ein praxisnaher Studiengang überhaupt Theorieelemente haben? Eine Antwort liegt auf der Hand. Das Lösungswissen in der WI veraltet sehr schnell. Technologien werden ständig weiterentwickelt, in allen Gebieten gibt es alle paar Jahre Technologiesprünge. Methodenwissen hat in der WI in Teilen nur eine recht kurze Halbwertszeit von ein paar Jahren, etwa wenn man an Managementmethoden denkt. Theoriewissen ist dagegen wesentlich stabiler und gibt damit wichtige Orientierungspunkte auch noch Jahrzehnte nach dem Studium.

Daneben ist aber auch zu berücksichtigen, dass die WI als universitäres Fach immer im Wettbewerb mit anderen universitären Disziplinen steht. Neben der Entwicklung und Vermittlung anwendungsnaher Lösungen sind die Entwicklung und Vermittlung von Theorien unstrittig konstituierendes Element einer Universität. Will die WI als universitäres Fach Anerkennung finden, dann darf sie sich einer Theorieorientierung nicht entziehen. Andere anwendungsnahe Fächer, wie die Ingenieurwissenschaften oder die Medizin, verzichten auf eine Theorieorientierung übrigens auch nicht, man denke nur an die Bedeutung physikalischer Grundlagen für die Ingenieurwissenschaften bzw. biologischer Grundlagen für die Medizin!

Als zweites stellt sich die Frage nach dem was, d. h. nach den relevanten Theorien für WI-Studiengänge. Dazu ist zunächst auf den interdisziplinären Charakter der WI und damit auf die Bezüge der WI, sowohl zu den Wirtschaftswissenschaften als auch zur Informatik, hinzuweisen. Eine erste Feststellung muss es daher sein, dass es keinesfalls ausreicht, wenn sich WI-Studiengänge nur den anwendungsnahen Themen aus den Wirtschaftswissenschaften und der Informatik annehmen. Vielmehr sollten die theoretischen Grundlagen der Wirtschaftswissenschaften (etwa in Gestalt der Mikroökonomie) und der Informatik (etwa in der Theorie zur Berechenbarkeit oder zur Gestaltung von Algorithmen) unverzichtbare Elemente eines Studiums der WI sein - ein Streichen wäre hier fatal für den universitären Charakter eines
Studiengangs. Zwei ganz unterschiedliche Beispiele mögen diese Überlegungen illustrieren. Ohne tiefgehende Kenntnisse der Organisationstheorie lässt sich das Phänomen des Outsourcings von IKTAufgaben nicht fundiert betrachten. Genauso führt Unkenntnis über die Grenzen der Berechenbarkeit zu falschen Einschätzungen bzgl. der Leistungsfähigkeit der IKT-gestützten Lösung von Optimierungsproblemen etwa in der Logistik.

Bleibt noch die Frage nach originären Theorien der WI. Diese Frage kann man empirisch und normativ angehen. Normativ würde man als Wirschaftsinformatiker reflexartig zunächst sagen, dass ein eigenständiges Fach wie die WI auch eigenständige Theorien benötigt. Praktisch ist dies bisher nicht so. Lim et al. (2009) haben vielmehr klar aufgezeigt, dass sich das Information System Research (ISR) typischerweise bei den Theorien der Wirtschaftswissenschaften bzw. den dahinter stehenden Theorien der Verhaltenswissenschaften bedient. Eine analoge Untersuchung für die WI würde wahrscheinlich die Informatik und ggf. die Mathematik als weitere Quellen für Theorien aufzeigen - an der grundlegenden Aussage würde dies aber nichts ändern. Gleiches gilt für die bekannten Ausnahmen, etwa in Gestalt der vom ISR und auch von der WI stark vorangetriebenen Akzeptanztheorie. Insgesamt bleibt damit die Aussage stehen, dass sich die WI im Regelfall der Theorien verwandter Felder bedient. Als Randbemerkung: Vielleicht ist das aber auch gar nicht so schlimm. Vielleicht misst sich der Beitrag der WI doch eher in dem Beitrag für die „Mutterdisziplinen“ und in der Brückenfunktion zwischen diesen.

Abschließend stellt sich die Frage nach dem wann, d. h. nach der Frage zu welchem Zeitpunkt Theorieelemente in die universitäre WI-Ausbildung einfließen sollen. Der typische universitäre Ansatz wäre es, mit der Vermittlung der Theorien zu beginnen und ergänzend aktuelle praktische Lösungen aufzuzeigen. Grundsätzlich gilt dies für die WI natürlich auch. Allerdings macht es die notwendige Breite des theoretischen $\mathrm{Zu}$ gangs über zwei Fächer schwer, diese Anforderung einzuhalten. Das pragmatische Ergebnis kann daher nur sein, am Anfang des Bachelorstudiums der WI selektiv theoretische Grundlagen aus den Wirtschaftswissenschaften und aus der Informatik zu vermitteln und die Theoriebezüge themenbezogen später $\mathrm{zu}$ ergänzen. Ein typisches Beispiel findet sich 
z. B. im Bereich der digitalen Güter. Die ökonomischen Charakteristika digitaler Güter sind sicherlich nicht Teil des Kanons zu den ökonomischen Grundlagen der WI, gleichwohl lassen sich digitale Produkte und Dienste aber etwa ohne tiefergehendes Verständnis von direkten oder indirekten Netzeffekten nicht wirklich verstehen.

Prof. Dr. Thomas Hess LMU München Sprecher der WKWI 2011-2013

\section{Merkmale und Profile von Wirtschaftsinformatikstudien- gängen an Universitäten und Fachhochschulen}

\subsection{Unterschiede und Gemeinsamkeiten}

Mit der Umstellung auf Bachelor- und Masterstudiengänge wurden die Curricula neu strukturiert. Die Wirtschaftsinformatik wird in unterschiedlichen Angebotsformen berücksichtigt. Von zentraler Bedeutung sind eigenständige Bachelorund Masterstudiengänge. Nachfolgend wird der eigenständige Bachelorstudiengang Wirtschaftsinformatik betrachtet, da er die zentrale berufsqualifizierende Ausbildung darstellt.

In Tab. 1 sind ausgewählte Merkmale von zwei zufällig ausgewählten Studiengängen (FH Münster und Universität zu Köln) dargestellt. Leider liegen derzeit keine verwendbaren CHE-Rankings vor, anhand derer sich eine Auswahl hätte orientieren können (CHE 2013). Die Auswahl erfolgte daher auf Basis einer Suchanfrage mit der Suchmaschine Google am 2013-04-04 und den Stichworten „studium wirtschaftsinformatik fh“ bzw. „studium wirtschaftsinformatik uni“. Ausgewählt wurden die ersten nicht gesponserten Links. WorkloadAspekte wurden aus Zeitgründen außen vor gelassen.

Die Lehrpläne sind ähnlich aufgebaut, die Unterschiede liegen im Detail. Die Gemeinsamkeiten sind vor allem in der Zielsetzung einer grundlagenorientierten Ausbildung für den Berufseinstieg und in formalen Aspekten (Studiendauer, Abschlussarbeit und Kolloquium) zu sehen. Grundsätzlich berücksichtigen beide Angebote zentrale Elemente der Informatik, der Betriebs- und Volkswirtschaftslehre, der Wirtschaftsinformatik sowie klassische Querschnittsthemen aus den Bereichen Mathematik, Statistik und Recht.
Daneben ist eine Thesis in Verbindung mit einem Kolloquium zu absolvieren. Das Wahlprogramm der Universität zu Köln ist differenzierter als das der FH Münster. Die Universität zu Köln bietet mit dem integrierten „Studium Integrale" und dem Wahlbereich die Möglichkeit der individuellen Profilbildung. Dafür sind die im FH-Studium obligatorischen Praxiselemente deutlich ausgeprägter.

Zum Vergleich wurden zwei weitere Beispiele ausgewählt (HTW Berlin und Universität Koblenz-Landau), die hier aus Platzgründen nicht wiedergegeben werden. Hierbei zeigen sich noch stärkere Ähnlichkeiten. Unterschiede sind nur noch in geringem Umfang erkennbar, beispielsweise wie zuvor in der Gestaltung der Praxisanteile. Die in beiden Fällen sichtbare Annäherung dürfte auch auf die „Rahmenempfehlung für die Universitätsausbildung in Wirtschaftsinformatik“ zurückzuführen sein (WKWI 2007), die auch die Lehrplangestaltung an Fachhochschulen stark geprägt hat.

\subsection{Notwendiges Praxiswissen und Theorie}

Die Wirtschaftsinformatikausbildung ist methodengeprägt. Schnelle Modenwechsel in der Praxis sorgen für rasche Veränderungen (Mertens 2006) auch in den Lehrplänen. Praxiswissen sollte nur zur exemplarischen Veranschaulichung und Überprüfung des Methodenwissens gelehrt werden. Generell ist Wert auf längerfristig nutzbares Wissen zu legen. Der Einsatz von Softwareprodukten sollte nicht im Vordergrund stehen.

\subsection{Profilierungsmöglichkeiten}

Die Spielräume für Differenzierungen der sich bereits annähernden Hochschultypen sind überschaubar, da beide $\mathrm{Ab}$ schlüsse zur Berufsfähigkeit führen.

Die Stärken der Fachhochschulen in der Anwendung, Adaption und anwendungsorientierten Weiterentwicklung von Methoden und Theorien sind auszubauen. Die kleineren Studierendengruppen tragen diesem Modell Rechnung. Die Universitäten können ihre Kompetenz der Entwicklung von grundlegenden Theorien und Methoden ausbauen. Überlappungen sind wünschenswert für die Durchlässigkeit. Keinesfalls darf der systemübergreifende Wechsel behindert werden.
Fachhochschulen können sich in praxisnahen Lehrveranstaltungen profilieren, bei denen die Methodenanwendung im Vordergrund steht. Universitäten können ihre Stärke der umfangreicheren Forschungsaktivitäten nutzen.

\subsection{Was erwartet der Arbeitsmarkt von den Absolventen?}

Die Absolventen sehen sich grundsätzlich den gleichen Anforderungen gegenübergestellt. Sie müssen in der Lage sein, in einem volatilen Umfeld selbständig anspruchsvolle technisch-organisatorische Probleme zu lösen. Hierzu benötigen sie solide theoretische Kenntnisse, aber auch die Fähigkeit, Methodenwissen auf konkrete Praxisprobleme anzuwenden. Die Anforderungen an Studieninhalte oder Hochschultypen werden in Stellenanzeigen oft breit gehalten, um die Anzahl der potenziellen Bewerber groß zu halten. Typische Formulierungen sind z. B. „Wir erwarten von Ihnen ein abgeschlossenes Studium der Informatik, Wirtschaftsinformatik, Mathematik oder ein abgeschlossenes Studium der Betriebs-/Volkswirtschaftslehre, Wirtschaftswissenschaften, Wirtschaftsingenieurwesen oder ähnlicher Studiengänge ..." (Abracon 2013). Die Wirtschaftsinformatik steht also im Wettbewerb mit anderen Abschlüssen. In den späteren Berufsjahren entscheiden persönlicher Einsatz, anwendungsorientierte Fachkenntnisse, Führungs- und Kommunikationsfähigkeiten sowie eine permanente Weiterbildung (z. B. in berufsbegleitenden Masterprogrammen) über den weiteren Erfolg.

\subsection{Fazit}

Die Stärken der Fachhochschulen liegen in der intensiven Verzahnung von Praxiselementen. Die Universitäten können Akzente in Richtung Forschung setzen.

Andreas Gadatsch Hochschule Bonn Rhein Sieg

\section{Merkmale und Profile von Wirtschaftsinformatikstudien- gängen an Universitäten und an Fachhochschulen}

Früher hatte man zwei Möglichkeiten: Universität oder Fachhochschule. An die Universität gingen diejenigen, die es lieber etwas fundierter haben wollten, für 
Tab. 1 Wirtschaftsinformatik-Bachelorstudiengänge (Fachhochschule Münster 2013; Universität zu Köln 2013)

\begin{tabular}{|c|c|c|}
\hline Hochschule & FH Münster (FH) & Universität zu Köln \\
\hline Abschluss & Bachelor of Science & Bachelor of Science \\
\hline Dauer & 6 Semester & 6 Semester \\
\hline \multirow[t]{2}{*}{ Zielsetzung } & Grundlagenvermittlung & Grundlagenvermittlung \\
\hline & $\begin{array}{l}\text { Direkteinstieg in den Beruf (z. B. branchenübergreifend } \\
\text { als Softwareentwickler, Analytiker, Berater) }\end{array}$ & $\begin{array}{l}\text { Direkteinstieg in Beruf (in verschiedenen Branchen mit } \\
\text { einer „Doppelqualifikation aus den Bereichen } \\
\text { Betriebswirtschaft und Informatik“ }\end{array}$ \\
\hline \multirow[t]{2}{*}{ BWL/VWL } & $\begin{array}{l}\text { Grundlagen der Betriebswirtschaftslehre, } \\
\text { Volkswirtschaftslehre }\end{array}$ & $\begin{array}{l}\text { Pflichtmodule: Bilanzen, Kosten- und Leistungsrechnung, } \\
\text { Technik des betrieblichen Rechnungswesens }\end{array}$ \\
\hline & Betriebswirtschaftliche Primärprozesse & $\begin{array}{l}\text { Wahlkatalog mit zahlreichen Fächern aus der BWL sowie } \\
\text { Mikroökonomik }\end{array}$ \\
\hline Informatik & $\begin{array}{l}\text { Betriebssysteme u. Rechnerarchitekturen, } \\
\text { Software-Entwicklung I/II, Web-Entwicklung I/II, } \\
\text { Rechnernetzwerke, Web-Engineering }\end{array}$ & $\begin{array}{l}\text { Informatik I/II, Programmierkurs, } \\
\text { Programmierpraktikum }\end{array}$ \\
\hline \multirow[t]{2}{*}{ Wirtschaftsinformatik } & $\begin{array}{l}\text { Grundlagen der Wirtschaftsinformatik, Grundlagen der } \\
\text { Programmierung }\end{array}$ & $\begin{array}{l}\text { Pflichtmodule: Integrated Information Systems, Database } \\
\text { Systems, Management of Information Systems Project }\end{array}$ \\
\hline & $\begin{array}{l}\text { Betriebliche Anwendungssysteme I/II, Business } \\
\text { Engineering, Business Intelligence }\end{array}$ & $\begin{array}{l}\text { Vier Wahlmodule: Systementwicklung, } \\
\text { Datenbanksysteme, Nutzung von Daten bei } \\
\text { Entscheidungsproblemen und Informationsmanagement } \\
\text { u.a. }\end{array}$ \\
\hline Querschnitt & $\begin{array}{l}\text { Wirtschaftsmathematik, Statistik, Wirtschaftsrecht, } \\
\text { Quantitative Methoden, Englisch }\end{array}$ & $\begin{array}{l}\text { Mathematik, Wahrscheinlichkeitsrechnung und Statistik, } \\
\text { Handels- und Gesellschaftsrecht (optional) }\end{array}$ \\
\hline Wahlpflicht & $\begin{array}{l}\text { Zwei Wahlpflichtfächer, Katalog mit } 8 \\
\text { Auswahlmöglichkeiten }\end{array}$ & $\begin{array}{l}\text { Sehr umfangreiches Angebot, auch aus } \\
\text { Nachbardisziplinen, spezielle Profilgruppen ermöglichen } \\
\text { individuelle Schwerpunkte, z. B. Finance, } \\
\text { Gesundheitsökonomie }\end{array}$ \\
\hline Abschlussarbeit & Bachelorarbeit mit Kolloquium & Bachelorarbeit mit Kolloquium \\
\hline Praxisanteile & $\begin{array}{l}\text { Praxisphasen von drei und sechs Monaten in der Endphase } \\
\text { des Studiums }\end{array}$ & Programmierpraktikum \\
\hline
\end{tabular}

die eventuell eine spätere Promotion in Betracht kam oder die vom Elternhaus in diese Richtung gelenkt wurden. An die Fachhochschule gingen diejenigen, die schnell und relativ sicher den Abschluss haben wollten und anschließend ohnehin vorhatten, direkt in die Praxis zu gehen.

Mit dem Bologna-Prozess ist die Entscheidungssituation für Studieninteressierte vielfältiger, aber nicht einfacher geworden. Universitäten wie Fachhochschulen bieten Bachelor- und Masterstudiengänge an, die beide berufsqualifizierend sein müssen. Der Zugang zu einem Masterstudiengang setzt einen berufsqualifizierenden Abschluss, in der Regel den Bachelor, voraus. Der Studierende wird in beiden Arten von Studiengängen eng geführt, er absolviert Module, die in der Regel über ein Semester gehen und mit ECTS-Punkten (ECTS $=$ European Credit Transfer and Accumulation System) als Volumenmaß und mit einer Note als Leistungsmaß versehen sind. Der Studierende kann sich nun entscheiden, ob er Bachelor und Master an einer der Hochschularten durchzieht, ob er nach dem Bachelor aufhört und dessen Berufsqualifizierung nutzt um in die Praxis zu gehen, ob er den Bachelor an der Fachhochschule macht und zum Master an die Universität geht oder umgekehrt. Promovieren kann er in jedem Fall, denn hier ist der Master der Fachhochschule dem der Universität aus formaler Sicht gleichgestellt. Die Ausgestaltung der Studiengänge enthält Freiheitsgrade, wie Bachelorstudiengänge mit 6 bis 8 und korrespondierend Masterstudiengänge mit 2 bis 4 Semestern. Es entstehen dabei einige zum Teil kuriose Varianten, wie z. B. „Fast Tracks“ für Promotionsstudierende.

Will man sich jenseits der politischen Willensbildung (die Fachhochschulen heißen mittlerweile Hochschulen für angewandte Wissenschaften (englisch: Universities of Applied Sciences) oder nur Hochschule, die Abschlussgrade lassen keinen Rückschluss auf den Hochschultyp zu) über den jeweiligen Auftrag informieren, so ist ein Blick in die Gesetzeslage hilfreich. Zum Beispiel formuliert das Bayerische Hochschulgesetz
(2012) in Artikel 2 (Aufgaben): „Die Universitäten dienen vornehmlich der Forschung und Lehre und verbinden diese $\mathrm{zu}$ einer vorwiegend wissenschaftsbezogenen Ausbildung“. Und andererseits: „Die Fachhochschulen vermitteln durch anwendungsbezogene Lehre eine Bildung, die zur selbstständigen Anwendung wissenschaftlicher Methoden und künstlerischer Tätigkeiten in der Berufspraxis befähigt; in diesem Rahmen führen sie anwendungsbezogene Forschungs- und Entwicklungsvorhaben durch". Wissenschaftsbezogene Ausbildung versus Bildung zur selbstständigen Anwendung wissenschaftlicher Methoden ist also der Unterschied im Auftrag an die beiden Arten von Hochschulen.

An dieser Stelle kommt das Fachgebiet Wirtschaftsinformatik ins Spiel. Nimmt man in der Wirtschaftsinformatik die Halbwertszeit für Theoriewissen mit 15 Jahren, die für Methodenwissen mit 5 Jahren und die für Produktwissen (nicht Praxiswissen!) mit 6 Monaten an und interpretiert den Auftrag an die Hochschularten wörtlich, so stellen sich folgende Fragen: 
- Wann lehrt man am besten die theoretischen Grundlagen der Wirtschaftsinformatik im Curriculum? Ab dem ersten Fachsemester! Hier werden die Strukturen des Faches gelegt und die grundlegenden Systematiken aufgebaut. Die Studierenden werden für das Fachgebiet geprägt.

- Warum gibt es keinen einstufigen Master in der Wirtschaftsinformatik? Das ist einer der Konstruktionsfehler bei der Implementierung des BolognaProzesses. Ein einstufiger Masterstudiengang würde das Problem einer wissenschaftsbezogenen (Grund-) Ausbildung lösen, das verkrampfte Suchen nach einer Berufsqualifizierung des Bachelor vermeiden, international verständlich sein und Studienzeit einsparen (u. a. Entfall der Bachelorarbeit, Wegfall von Friktionen beim Übergang zwischen Bachelor und Master).

- Haben es diejenigen, die den Bachelor an der Fachhochschule erworben haben, in einem universitären MasterStudiengang Wirtschaftsinformatik nicht unverhältnismäßig schwer? Ja, im Grunde schon. Aber immerhin sind es die motiviertesten Bachelorabsolventinnen und -absolventen, die zum Masterstudium an die Universität gehen, und weder die Fachhochschulen noch die Universitäten verhalten sich im Allgemeinen idealtypisch nach ihrem Auftrag. Trotzdem wurden z. B. an der Universität Bamberg für diesen Fall Vorkehrungen getroffen, indem man in begründeten Fällen und auf Antrag max. 12 ECTS-Punkte aus dem Bachelorprogramm in das Masterprogramm einbringen kann.
Wir bereiten heute unsere Absolventinnen und Absolventen auf eine 44jährige Berufstätigkeit vor (18 Jahre bis zum Abitur, 5 Jahre Studium, 67 Jahre Renteneintritt). Dabei wissen wir nicht, was in einem dynamischen Fachgebiet wie der Wirtschaftsinformatik in 5 und in 10 Jahren auf uns zukommt. Wäre es da nicht angezeigt, möglichst viele junge Leute wissenschaftsbezogen auszubilden und damit in die Lage zu versetzen, sich autonom auf Unbekanntes einstellen und Neues gestalten zu können? „Nichts ist so praktisch wie eine gute Theorie“, dieser Satz des Sozialpsychologen Kurt Lewin hat auch heute seine unverminderte Bedeutung.

Prof. Dr. Elmar J. Sinz ${ }^{1}$ Universität Bamberg

\section{Literatur}

\section{zu: Abschnitt 1}

Loos $\mathrm{P}$, Mertens $\mathrm{P}$, Eymann T, Hirschheim R, Schwenker B, Hess T (2013) Qualification profile of university professors in business and information systems engineering. Bus Inf Syst Eng 5(2):107-114

\section{zu: Abschnitt 3}

Laudon JP, Laudon KC (2002) Management information systems: managing the digital firm, 7th edn. Prentice Hall, Upper Saddle River

Laudon JP, Laudon KC, Schoder D (2009) Wirtschaftsinformatik: Eine Einführung, 2. Aufl. Pearson, München

\section{zu: Abschnitt 4}

Lim S, Saldanha T, Malladi S, Melville NP (2009) Theories used in information systems research: identifying theory networks in leading IS journals. In: Proc ICIS 2009, Phoenix. paper 91

\section{zu: Abschnitt 5}

Abracon $\mathrm{GmbH}$ (2013) Stellenangebot SAP NetWeaver BI/BW Juniorberater ( $\mathrm{w} / \mathrm{m})$. http://www.abracon.de/sap-netweaver-bibw-juniorberater-wm.html. Abruf am 2013-04-02

CHE (2013) CHE Hochschulranking 2012/13. http://ranking.zeit.de/che2012/de/

fachinfo?esb=2. Abruf am 2013-04-04

Fachhochschule Münster (2013). Wirtschaftsinformatik (Bachelor). https://www. fh-muenster.de/studium/studiengaenge/ bachelorstudiengaenge.php. Abruf am 2013-04-04

Mertens P (2006) Moden und Nachhaltigkeit in der Wirtschaftsinformatik. Arbeitspapier Nr 1, 2006, Universität Nürnberg-Erlangen. http://www.wi1-mertens.wiso.unierlangen.de/veroeffentlichungen/suche. php?typ=arbeitsberichtwii\&order $1=$ autoren\&ascdes $c 1=$ asc\&order $2=$ titel\&ascdesc2=asc. Abruf am 2013-03-21

Universität zu Köln (2013) Wirtschaftsinformatik (Bachelor). http://www.wiso.unikoeln.de/14714.html. Abruf am 2013-0404

WKWI (2007) Rahmenempfehlung für die Universitätsausbildung in Wirtschaftsinformatik. Von einer Fachkommission im Auftrag der Wissenschaftlichen Kommission (WK) Wirtschaftsinformatik im Verband der Hochschullehrer für Betriebswirtschaft erarbeitet; von der WK am 1.3.2007 und vom Präsidium der Gesellschaft für Informatik am 28.6.2007 genehmigt pp 2013-03-2013-21. http://fbwi.gi.de/fileadmin/gliederungen/fb-wi/wiempf-2007.pdf. Abruf am 2013-03-21

\section{zu: Abschnitt 6}

Bayerisches Hochschulgesetz (2012) vom 23. Mai 2006, zuletzt geändert am 9. Juli 2012

\footnotetext{
${ }^{1}$ Der Autor leitete acht Jahre den Fachausschuss Wirtschaftsinformatik bei der Akkreditierungsagentur ASIIN und war acht Jahre Mitglied des Fachausschusses Informatik+ bei ACQUIN.
} 